\title{
Estudo comportamental sobre a drenagem axilar no câncer de mama
}

ARTIGO ORIGINAL

\section{Descritores}

Câncer de mama

Seroma

Drenagem

\section{Behavioral study of the axillary drainage in breast cancer}

Ruffo Freitas-Junior ${ }^{1}$, Ana Flávia Ribeiro Santos Cavalcante², Leonardo Ribeiro Soares ${ }^{3}$, Ana Paula Queiroz Pádua ${ }^{4}$, Patrícia Tavares Pereira Sousa ${ }^{5}$, Luís Fernando Jubé Ribeiro ${ }^{6}$, Márcia Faria Veloso ${ }^{7}$

\section{RESUMO}

Objetivo: Avaliar os sentimentos e as dificuldades vivenciadas pelos pacientes durante a utilização de drenos de sucção. Métodos: Trata-se de um estudo descritivo, transversal, que avaliou mulheres portadoras de câncer de mama submetidas ao tratamento cirúrgico e que permaneceram com dreno de sucção por três dias, no mínimo. Foi avaliada a dificuldade para a realização de atividades diárias, gerais e específicas, assim como os sentimentos negativos desencadeados pela utilização do dreno, por intermédio da aplicação de questionário previamente estabelecido. Resultados: Foram incluídas no estudo 77 pacientes, com média de idade de $49( \pm 13)$ anos. Entre a população analisada, 53 pacientes $(68,8 \%)$ deixaram de realizar alguma tarefa por estar com o dreno. Entre as limitaçóes, destacam-se as dificuldades para "vestir-se" (79,2\%) e para "dormir" (87,0\%). Entre os sentimentos referidos, encontrou-se prevalência de "estranheza" (76,6\%) e "medo" (72,7\%). Conclusáo: Em posse dos resultados observados, o cirurgião deverá considerar para a decisão de utilizar drenos após manipulação axilar em cirurgias mamárias, de um lado, o manejo de serosidades e, de outro, as dificuldades diárias e os sentimentos negativos vivenciados pelos pacientes com a utilização desses sistemas de drenagem.

\section{ABSTRACT}

Objective: To evaluate the feelings and the difficulties experienced by the patients during the period when they are using suction drain. Methods: This transversal, descriptive study evaluated women with breast cancer, submitted to surgical treatment and who used the suction drain for at least three days. The difficulties to perform specific and general daily tasks, as well as the negative feelings triggered by the drain were evaluated by means of a questionnaire specifically designed for this study. Results: 77 patients, with the mean age of 49 ( \pm 13 years). Among the sample, 53 (68.8\%) of the studied patients stopped performing certain tasks because they were using the drain. Regarding limitations, the difficulty to get dressed (79.2\%) and to sleep (87.0\%) are the highlighted ones. Among the feelings reported there was a prevalence of "strangeness" (76.6\%) and "fear" (72.7\%). Conclusion: Having these results in mind, when the surgeon considers using the drain after axillary manipulation in breast surgery, they must take into account on one hand the handling of seroma, and on the other hand, the daily difficulties and the negative feelings experienced by the patients during the use of drains.

Trabalho realizado na Rede Goiana de Pesquisa em Mastologia - Goiânia (G0), Brasil.

PProfessor Adjunto e Coordenador do Programa de Mastologia da Universidade Federal de Goiás (UFG); Médico Titular do Hospital Araújo Jorge da Associação de Combate ao Câncer em Goiás (ACCG) - Goiânia (GO), Brasil.

${ }^{2}$ Médica do Hospital da Mulher e Maternidade Dona Íris - Goiânia (GO), Brasil.

${ }^{3}$ Médico Residente de Ginecologia e Obstetrícia da UFG - Goiânia (GO), Brasil.

${ }^{4}$ Médica do Hospital da Mulher e Maternidade Dona Íris - Goiânia (GO), Brasil.

${ }^{5}$ Médica do Instituto Goiano de Medicina (IGM) - Goiânia (GO), Brasil.

${ }^{6}$ Médico Titular do Hospital Araújo Jorge da ACCG - Goiânia (G0), Brasil.

${ }^{7}$ Psicóloga do Programa de Mastologia da UFG - Goiânia (GO), Brasil.

Endereço para correspondência: Ruffo Freitas-Junior - Programa de Mastologia do Hospital das Clínicas da Universidade Federal de Goiás -

$1^{a}$ Avenida, s/n - Setor Universitário - CEP: 74605-050 - Goiânia (G0), Brasil - E-mail: ruffojr@terra.com.br

Conflito de interesses: nada a declarar.

Recebido em: 23/01/2015. Aceito em: 26/01/2015 


\section{Introdução}

O câncer de mama representa um problema de saúde pública, sendo a neoplasia de maior incidência na população feminina brasileira ${ }^{1-3}$. Nos últimos anos, a consolidação dos programas de rastreamento ${ }^{4-6}$ e de novos modelos diagnósticos ${ }^{7-9}$ tem permitido uma mudança na evoluçáo temporal do câncer de mama no Brasil, com reduçáo dos casos avançados e aumento do diagnóstico dos casos iniciais ${ }^{10}$.

O diagnóstico precoce é o principal responsável pelos avanços no tratamento cirúrgico do câncer de mama, permitindo a investigação do linfonodo sentinela e a realização de técnicas cirúrgicas de conservação mamáriaa ${ }^{11-13}$. Apesar dos benefícios com os resultados estéticos, as taxas de morbidade pós-operatória permanecem elevadas, tanto com as técnicas conservadoras quanto com o tratamento radical ${ }^{12,14-16}$.

Entre as complicaçôes pós-operatórias do câncer de mama, a formação de seroma permanece como a mais frequente, apresentando incidência que varia de 3 a $85 \%{ }^{17,18}$. Além disso, o seroma também está associado a necrose cutânea, dificuldades para a cicatrizaçáo da ferida operatória, infecção local e linfedema de membro superior ${ }^{18-20}$.

Nessas situaçôes, a abordagem do seroma inclui a utilização de diferentes sistemas de drenagem, que geralmente são inseridos de forma profilática durante o procedimento cirúrgico. Apesar das divergências envolvendo o tipo de drenagem, o tempo de permanência e o uso de antibióticos ${ }^{18-20}$, pouco se sabe sobre as repercussôes psicossociais envolvendo o método. Assim, este estudo teve como objetivo a avaliação dos sentimentos e das dificuldades vivenciadas pelas pacientes durante a utilização de drenos de sucção.

\section{Metodologia}

Trata-se de um estudo descritivo, transversal, de abordagem quantitativa, realizado em dois serviços de referência para o diagnóstico e o tratamento do câncer de mama localizados na cidade de Goiânia (GO), Brasil.

$\mathrm{O}$ estudo foi realizado com uma amostra de conveniência constituída por mulheres portadoras de câncer de mama submetidas ao tratamento cirúrgico no período de janeiro de 2005 a dezembro de 2006 e que permaneceram com dreno de sucção por três dias, no mínimo. Também foram seguidos os seguintes critérios de inclusão: idade superior a 18 anos, capacidade de compreensão das orientaçóes fornecidas e condiçôes físicas para a manipulação individual dos sistemas de drenagem.

Foi aplicado um questionário específico, desenvolvido em parceria com o Serviço de Psicologia do Programa de Mastologia do Hospital das Clínicas da Universidade Federal de Goiás (HC/UFG). Os questionários foram preenchidos pelos próprios entrevistadores, entre o $3^{\circ}$ e o $10^{\circ}$ dia de pós-operatório, após aplicação do Termo de Consentimento Livre e Esclarecido.

Inicialmente, as pacientes incluídas no estudo responderam as seguintes perguntas: "Você sabe por que está utilizando esse dreno?" e "Você deixou de realizar alguma tarefa por estar com o dreno?". Posteriormente, foi avaliada a dificuldade para a realização de atividades diárias, gerais e específicas, em decorrência da utilização do dreno, como, por exemplo, "vestir-se", "mexer o braço" e "caminhar". Essas variáveis foram categorizadas de acordo com o grau de intensidade da limitação: "nada", "um pouco", "bastante" e "muitíssimo". Também foram avaliados os sentimentos negativos desencadeados pela utilização do dreno, como, por exemplo, "vergonha", "insegurança" e "medo", os quais também foram categorizados de acordo com o grau de intensidade.

As informaçōes coletadas foram transcritas para um banco de dados específico, desenvolvido com o auxílio do programa Microsoft Office Excel ${ }^{\circledR}$, versão 2003 (Microsoft, EUA). Após compilação e checagem das informaçôes, foi realizada análise descritiva por meio de distribuição de frequências.

O estudo atual faz parte de uma linha de pesquisa desenvolvida pela Rede Goiana de Pesquisa em Mastologia, com aprovaçáo pelo Comitê de Ética em Pesquisa do HC/UFG.

\section{Resultados}

Foram incluídas no estudo 77 pacientes, com média de idade de $49( \pm 13)$ anos. Os dados demográficos estâo apresentados na Tabela 1 e mostram prevalência de mulheres casadas $(62,3 \%)$, de religião católica $(54,5 \%)$, residentes no interior do Estado de Goiás (54,5\%) e com grau de instruçâo primário $(53,2 \%)$. Em relação ao tratamento cirúrgico, 37 pacientes $(48,0 \%)$ foram submetidas ao tratamento com conservação mamária (quadrantectomia), e 40 pacientes $(52,0 \%)$, à mastectomia.

Entre a população analisada, 55 pacientes $(71,4 \%)$ desconheciam o motivo pelo qual estavam utilizando o sistema de drenagem. Além disso, 53 pacientes $(68,8 \%)$ deixaram de realizar alguma tarefa por estar com o dreno.

A Tabela 2 evidencia as limitaçôes que as pacientes enfrentaram em diferentes situaçóes por estar com o dreno, incluindo as dificuldades para "vestir-se" (79,2\%), para "realizar tarefas diárias" (77,9\%), bem como para "dormir” (87,0\%).

Já a Tabela 3 evidencia os sentimentos referidos pelas pacientes durante a utilização de drenos, no pós-operatório de cirurgias para o tratamento do câncer de mama, mostrando prevalência de "estranheza" (76,6\%), de "medo" (72,7\%) e de "insegurança”" (71,4\%). 
Tabela 1. Distribuição das características sociodemográficas da população analisada $(\mathrm{n}=77)$

\begin{tabular}{lcc}
\hline Variáveis & $\mathbf{n}$ & $\%$ \\
Estado civil & 9 & \\
$\quad$ Solteira & 48 & 62,3 \\
Casada & 13 & 16,9 \\
Viúva & 7 & 09,1 \\
Divorciada & & \\
Grau de instrução & 15 & 19,5 \\
Analfabeta & 41 & 53,2 \\
Ensino fundamental & 19 & 24,7 \\
Ensino médio & 2 & 2,6 \\
Ensino superior & & \\
Religião & 42 & 54,5 \\
Católica & 29 & 37,7 \\
Evangélica & 3 & 3,9 \\
Espírita & 1 & 1,3 \\
Ausente & 2 & 2,6 \\
Outras & & \\
Residência & & \\
Goiânia & 18 & 23,4 \\
Interior de Goiás & 42 & 54,5 \\
Outros Estados & 17 & 22,1 \\
Cirurgia & & \\
Quadrantectomia & 37 & 48,0 \\
Mastectomia sem reconstrução & 50,7 \\
Mastectomia com reconstrução & 1,3 \\
\hline
\end{tabular}

\section{Discussão}

Os sistemas de drenagem representam o principal método no manejo dos seromas relacionados a cirurgias mamárias, ainda que pese a associação entre utilização dos drenos e maior taxa de infecção local, entre outros desfechos negati$\operatorname{vos}^{20,21}$. Nesse contexto, diversos estudos avaliaram a redução das complicaçôes cirúrgicas por intermédio de uma abordagem menos agressiva, incluindo as técnicas de investigação do linfonodo sentinela ${ }^{12,13,22}$. Tais técnicas, associadas à redução das taxas de linfadenectomias, contribuíram para a redução das complicaçóes pós-operatórias relacionadas à abordagem axilar, em especial para as pacientes obesas, que apresentam maior incidência de deiscência e de formação de seromas ${ }^{18,23}$. No estudo atual, todas as pacientes foram submetidas à linfadenectomia. Portanto, as dificuldades e os sentimentos relacionados aos sistemas de drenagem devem ser fonte de novos estudos, em vista dos recentes avanços na investigação axilar do câncer de mama e na esperada redução das complicações cirúrgicas.

A movimentação cuidadosa e precoce do braço ipsilateral após a cirurgia está associada a melhor resolução de seromas, com menor número de punçóes e menor volume puncionado ${ }^{24}$. No entanto, a mobilidade exagerada do membro pode contribuir para a maior incidência de hematomas e seromas ${ }^{18}$.

Tabela 2. Dificuldades relacionadas à utilização de sistemas de drenagem vivenciadas por portadoras de câncer de mama submetidas ao tratamento cirúrgico

\begin{tabular}{lcccccccc}
\hline \multirow{2}{*}{ Atrapalha } & \multicolumn{2}{c}{ Não } & \multicolumn{2}{c}{ Um pouco } & \multicolumn{2}{c}{ Bastante } & \multicolumn{2}{c}{ Muitíssimo } \\
\cline { 2 - 8 } & $\mathbf{n}$ & $\%$ & $\mathbf{n}$ & $\%$ & $\mathbf{n}$ & $\%$ & $\mathbf{n}$ & $\%$ \\
Vestir-se & 16 & 20,8 & 35 & 45,5 & 9 & 11,7 & 17 & 22,1 \\
Comer & 67 & 87,0 & 7 & 9,1 & 2 & 2,6 & 1 & 1,3 \\
Sentar-se & 57 & 74,0 & 13 & 16,9 & 6 & 7,8 & 1 & 1,3 \\
Higiene diária & 20 & 26,0 & 27 & 35,1 & 19 & 24,7 & 11 & 14,3 \\
Sair em público & 36 & 46,8 & 14 & 18,2 & 12 & 15,6 & 15 \\
Mexer o braço & 29 & 37,7 & 24 & 31,2 & 12 & 15,6 & 12 & 15,5 \\
Caminhar & 57 & 74,0 & 17 & 22,1 & 3 & 3,9 & 0 & 0 \\
Realizar tarefas diárias & 17 & 22,1 & 21 & 27,3 & 20 & 26,0 & 19 & 24,7 \\
Dormir & 10 & 13,0 & 18 & 23,4 & 19 & 24,7 & 30 \\
\hline
\end{tabular}

Tabela 3. Sentimentos relacionados à utilização de sistemas de drenagem vivenciados por portadoras de câncer de mama submetidas ao tratamento cirúrgico

\begin{tabular}{lcccccccc}
\hline \multirow{2}{*}{ Sentimentos } & \multicolumn{2}{c}{ Não } & \multicolumn{2}{c}{ Um pouco } & \multicolumn{2}{c}{ Bastante } & \multicolumn{2}{c}{ Muitíssimo } \\
\cline { 2 - 9 } & $\mathbf{n}$ & $\%$ & $\mathbf{n}$ & $\%$ & $\mathbf{n}$ & $\%$ & $\mathbf{n}$ & $\%$ \\
Vergonha & 56 & 72,7 & 10 & 13,0 & 6 & 7,8 & 5 & 6,5 \\
Nojo & 54 & 70,1 & 10 & 13,0 & 5 & 6,5 & 8 & 10,4 \\
Irritaçáo & 47 & 61,0 & 15 & 19,5 & 8 & 10,4 & 7 & 9,1 \\
Raiva & 59 & 76,6 & 10 & 13,0 & 3 & 3,9 & 5 & 6,5 \\
Insegurança & 22 & 28,6 & 20 & 26,0 & 16 & 20,8 & 19 & 24,7 \\
Tristeza & 50 & 64,9 & 14 & 18,2 & 8 & 10,4 & 5 & 6,5 \\
Medo & 21 & 27,3 & 20 & 26,0 & 16 & 20,8 & 20 & 26,0 \\
Invasáo & 37 & 48,1 & 20 & 26,0 & 15 & 19,5 & 5 & 6,5 \\
Estranheza & 18 & 23,4 & 22 & 28,6 & 22 & 28,6 & 15 & 19,5 \\
Dor & 25 & 32,5 & 34 & 44,2 & 7 & 9,1 & 11 & 14,3 \\
\hline
\end{tabular}


No atual estudo, 48 pacientes $(62,3 \%)$ relataram algum grau de dificuldade para "mexer o braço" durante a utilização do dreno, eventualmente podendo comprometer o manejo dos seromas, assim como gerar dificuldade na realização adequada da radioterapia.

A limitação para realizar tarefas diárias, como "dormir" e "vestir-se", pode interferir de forma negativa na evoluçâo do processo saúde-doença, comprometendo a motivação e até mesmo a aderência ao tratamento médico proposto. Essas dificuldades também podem reduzir a qualidade de vida no período pós-operatório, conforme evidenciado por outros estudos, em situaçôes semelhantes ${ }^{21,25}$.

Outro aspecto psicossocial comprometido durante o tratamento do câncer de mama é a percepção feminina acerca da imagem corporal, o que pode gerar redução da autoestima, complexo de inferioridade e medo de rejeição por parte do parceiro $^{26}$. Acredita-se que os sistemas de drenagem, principalmente nos casos de permanência prolongada, possam interferir de forma negativa na autoimagem das pacientes, e, consequentemente, no seu posicionamento como mulher diante da sociedade.

Em relação aos sentimentos negativos, evidenciou-se no presente estudo a prevalência de "estranheza", "medo" e "insegurança”. Da mesma forma, representaçôes pessoais de "dor", "raiva", "nojo" e "vergonha" também foram associadas à utilização dos drenos, o que reforça a relevância do suporte psicológico aos portadores de câncer de mama. Esses sentimentos têm sido negligenciados pelos recentes estudos sobre a utilização de drenos após cirurgias mamárias, que priorizam os aspectos físicos em detrimento aos aspectos biopsicossociais.

Até onde sabemos, este é o primeiro estudo que quantificou a prevalência dos sentimentos vivenciados pelos pacientes durante a utilização de sistemas de drenagem, com avaliação dos diferentes graus de intensidade apresentados. Em posse dos resultados observados, o cirurgião deverá considerar para a decisão de utilizar drenos após manipulação axilar em cirurgias mamárias, de um lado, o manejo de serosidades e, de outro, as dificuldades diárias e os sentimentos negativos vivenciados pelos pacientes durante a utilização desses sistemas de drenagem.

\section{Referências}

1. Freitas-Junior R, Gonzaga CMR, Freitas NMA, Martins E, Dardes RCM. Disparities in female breast cancer mortality rates in Brazil between 1980 and 2009. Clinics. 2012;67(7):731-7.

2. Gonzaga CMR, Freitas-Junior R, Souza MR, Curado MP, Freitas NMA. Disparities in female breast cancer mortality rates between urban centers and rural areas of Brazil: Ecological time-series study. The Breast. 2014;23(2):180-7.

3. Brasil. Ministério da Saúde. Secretaria de Atenção à Saúde. Instituto Nacional de Câncer. Coordenação de Prevenção e Vigilância
[Internet]. Estimativa 2014: incidência de câncer no Brasil. Rio de Janeiro: INCA; 2014 [cited 2014 Dez 20]. Available from: http:// wwwl.inca.gov.br/estimativa/2014/

4. Corrêa RS, Freitas-Junior R, Peixoto JE, Rodrigues DCN, Lemos MEF, Martins LAP, et al. Estimativas da cobertura mamográfica no Estado de Goiás, Brasil. Cad Saúde Pública. 2011;27(9):1757-67.

5. Corrêa RS, Freitas-Junior R, Peixoto JE, Rodrigues DCN, Lemos MEF, Dias CM, et al. Efetividade de programa de controle de qualidade em mamografia para o Sistema Único de Saúde. Rev Saúde Pública. 2012;46:769-76.

6. van Luijt PA, Fracheboud J, Heijnsdijk EA, den Heeten GJ, de Koning HJ; National Evaluation Team for Breast Cancer Screening in Netherlands Study Group (NETB). Nation-wide data on screening performance during the transition to digital mammography: observations in 6 million screens. Eur J Cancer. 2013;49(16):3517-25.

7. Unger-Saldaña K. Challenges to the early diagnosis and treatment of breast cancer in developing countries. World J Clin Oncol. 2014;5(3):465-77.

8. Minamimoto R, Senda M, Jinnouchi S, Terauchi T, Yoshida $\mathrm{T}$, Inoue T. Detection of Breast Cancer in an FDG-PET Cancer Screening Program: Results of a Nationwide Japanese Survey. Clin Breast Cancer. 2014 [Epub ahead of print].

9. Paulinelli RR, Freitas-Junior R, de Lucena CE, Moreira MA, de Moraes VA, Bernardes-Júnior JR, et al. Sonobreast: predicting individualized probabilities of malignancy in solid breast masses with echographic expression. Breast J. 2011;17(2):152-9.

10. Nunes RD, Martins E, Freitas-Junior R, Curado MP, Freitas NMA, Oliveira JC. Estudo descritivo dos casos de câncer de mama em Goiânia, entre 1989 e 2003. Rev Col Bras Cir. 2011;38(4):212-6.

11. Veronesi U, Cascinelli N, Mariani L, Greco M, Saccozzi R, Luini A, et al. Twenty-year follow-up of a randomized study comparing breast-conserving surgery with radical mastectomy for early breast cancer. N Engl J Med. 2002;347(16):1227-32.

12. Donker M, van Tienhoven G, Straver ME, Meijnen P, van de Velde CJ, Mansel RE, et al. Radiotherapy or surgery of the axilla after a positive sentinel node in breast cancer (EORTC 10981-22023 AMAROS): a randomised, multicentre, open-label, phase 3 noninferiority trial. Lancet Oncol. 2014;15(12):1303-10.

13. Paulinelli RR, de Oliveira VM, Bagnoli F, Chade MC, Alves KL, Freitas-Junior R. Oncoplastic mammaplasty with geometric compensation-a technique for breast conservation. J Surg Oncol. 2014 Aug 13 [Epub ahead of print].

14. Blumenschein AR, Freitas-Junior R, Tuffanin AT, Blumenschein DI. Lipoenxertia nas mamas: procedimento consagrado ou experimental? Rev Bras Cir Plást. 2012;27(4):616-22.

15. Valinote SPA, Freitas-Junior R, Martins KA, Pereira AC, Pereira $\mathrm{CE}$, Martins E. Alteraçōes venosas e linfáticas em mulheres com linfedema após linfadenectomia axilar no tratamento do câncer de mama. Rev Bras Ginecol Obstet. 2013;35(4):171-7.

16. Mastrella AS, Freitas-Junior R, Paulinelli RR, Soares LR. Incidence and risk factors for winged scapula after surgical treatment for breast cancer. J Clin Nurs. 2014;23(17-18):2525-31.

17. Kumar S, Lal B, Misra MC. Post-mastectomy seroma: a new look into the aetiology of an old problem. J R Coll Surg Edinb. 1995;40(5):292-4.

18. Srivastava V, Basu S, Shukla VK. Seroma formation after breast cancer surgery: what we have learned in the last two decades. J Breast Cancer. 2012;15(4):373-80. 
19. Degnim AC, Hoskin TL, Brahmbhatt RD, Warren-Peled A, Loprinzi M, Pavey ES, et al. Randomized trial of drain antisepsis after mastectomy and immediate prosthetic breast reconstruction. Ann Surg Oncol. 2014;21(10):3240-8.

20. Okada N, Narita Y, Takada M, Kato H, Ambo Y, Nakamura F, et al. Early removal of drains and the incidence of seroma after breast surgery. Breast Cancer. 2015;22(1):79-83.

21. Thomson DR, Sadideen H, Furniss D. Wound drainage after axillary dissection for carcinoma of the breast. Cochrane Database Syst Rev. 2013;10:CD006823.

22. Andeweg CS, Schriek MJ, Heisterkamp J, Roukema JA. Seroma formation in two cohorts after axillary lymph node dissection in breast cancer surgery: does timing of drain removal matter? Breast J. 2011;17(4):359-64
23. Pan XF, Huan JL, Qin XJ. Potential risk factors for the development of seroma following mastectomy with axillary dissection. Mol Clin Oncol. 2015;3(1):222-6.

24. Gozzo TO, Almeida AM, Sanches MP, Brito LGO, Carrara HHA. Estudo comparativo da eficácia da movimentaçáo precoce do braço no controle do seroma pós-linfadenectomia axilar em mulheres com câncer de mama. Rev Bras Mastol. 2008;18(2):58-62.

25. Yuste-Sánchez MJ, Lacomba MT, Sánchez BS, Merino DP, da Costa SP, Téllez EC, et al. Health related quality of life improvement in breast cancer patients: Secondary outcome from a simple blinded, randomised clinical trial. Breast. 2014 Dec 10 [Epub ahead of print].

26. Prates ACL, Zanini DS, Veloso MF. Investimento corporal e o funcionamento sexual em mulheres no pós-cirúrgico de câncer de mama. Rev SBPH. 2012;15(1):264-78. 